Artículo científico

(Original paper)

\title{
ÁCAROS ASOCIADOS A NIDOS DE ÁGUILA REAL (AQUILA CHRYSAETOS CANADENSIS) (FALCONIFORMES: ACCIPITRIDAE) EN MÉXICO
}

\author{
MITES ASSOCIATED TO NESTS OF THE GOLDEN EAGLE (AQUILA CHRYSAETOS \\ CANADENSIS) (FALCONIFORMES: ACCIPITRIDAE) IN MEXICO
}

\begin{abstract}
MARía de JeSÚS DAMIÁN CHÁVEZ ${ }^{*}$, GABRIEL A. VILLEGAS GUZMÁN ${ }^{1,2}$, LUIS FELIPE LOZANO ROMÁN ${ }^{3}$

${ }^{1}$ Laboratorio de Acarología, Departamento de Zoología, Escuela Nacional de Ciencias Biológicas, Instituto Politécnico Nacional, Prolongación de Carpio y Plan de Ayala s/n, Col. Santo Tomás, C. P. 11340, México D. F. <mjedc_9@ yahoo.com.mx> ${ }^{2}$ Laboratorio de Conservación de Fauna Silvestre, Universidad Autónoma Metropolitana Iztapalapa, San Rafael Atlixco No. 186, Col. Vicentina, C. P. 09340, Delegación Iztapalapa, Ciudad de México. <gabrvill@yahoo.com>

${ }^{3}$ Comisión Nacional de Áreas Naturales Protegidas, Dirección de Especies Prioritarias para la Conservación. Avenida Ejército Nacional No. 223, Col. Anáhuac, I Sección, Del. Miguel Hidalgo, C. P. 11320, Ciudad de México.<flozano@conanp.gob.mx>

*Autor de correspondencia:<mjedc_9@yahoo.com.mx>
\end{abstract}

Recibido: 11/01/2018; aceptado: 24/01/2018; publicado en línea: 29/03/2019

Editor responsable: Guillermo Ibarra Núñez

Damián-Chávez, M. J., Villegas-Guzmán, G. A., Lozano-Román, L. F. (2019) Ácaros asociados a nidos de águila real (Aquila chrysaetos canadensis) (Falconiformes: Accipitridae) en México. Acta Zoológica Mexicana (nueva serie), 35, 1-16. https://doi.org/10.21829/azm.2019.3502196

RESUMEN. Los nidos de las aves pueden llegar a albergar a una gran diversidad de artrópodos, la cual está en función de la composición, estructura y tamaño del nido, así como de las actividades del ave. El componente más abundante de la artropodofauna presente en los nidos son los ácaros, los cuales comprenden la fauna asociada con el microambiente del nido. El objetivo del presente trabajo fue explorar la riqueza y diversidad de ácaros asociados a los nidos de águila real y aportar información sobre la asociación ácaros-nido. Se recolectaron dos nidos de águila real (Aquila chysaetos canadensis) el 19 de julio de 2013 en Sierra Fría, Zacatecas (Nido I) y Juan Grande, Aguascalientes (Nido II), México. Los materiales de los nidos se procesaron de acuerdo con la técnica de embudos de Berlese-Tullgren. Se encontraron 2,141 ácaros, pertenecientes a 68 especies de 49 familias para ambos nidos. Las hembras presentaron la mayor abundancia, con valores de $85.57 \%$ para el Nido I y $74.30 \%$ para el Nido II. Para el Nido I, la especie más abundante fue Macrocheles mexicanus (Evans \& Hyatt, 1963) (Macrochelidae), con un valor del $50.64 \%$, siendo las hembras más abundantes que los machos. Por otro lado, la especie más abundante en el Nido II fue Eulohmannia sp. (13.61\%), registrando hembras, deutoninfas, protoninfas y larvas. Se calculó la diversidad y riqueza para los dos nidos de águila real, donde el Nido II, presentó una mayor diversidad y riqueza de especies $\left(1-\mathrm{D}=0.927 ; D_{M g}=7.455\right)$. Este trabajo representa un primer acercamiento al conocimiento de la acarofauna asociada a nidos de águila real.

Palabras clave: Nidos; ácaros; asociación; abundancia; riqueza 
Damián-Chávez, M. J., Villegas-Guzmán, G. A., Lozano-Román, L. F. (2019) Mites associated to nests of the golden eagle (Aquila chrysaetos canadensis) (Falconiformes: Accipitridae) in Mexico. Acta Zoológica Mexicana (nueva serie), 35, 1-16. https://doi.org/10.21829/azm.2019.3502196

\begin{abstract}
The nests of the birds can host a great diversity of arthropods, which is a function of the composition, structure and size of the nest, as well as the activities of the bird. The most abundant component of the arthropod fauna present in the nests are the mites, which include the fauna associated with the microenvironment of the nest. The aim of the present work was to explore the richness and diversity of mites associated with the golden eagle nests and provide information on the mite-nest association. Two golden eagle nests (Aquila chysaetos canadensis) were collected on July 19, 2013 in Sierra Fría, Zacatecas (Nest I) and Juan Grande, Aguascalientes (Nest II), Mexico. All the materials of the nests were processed according to the Berlese-Tullgren funnel technique. For both nests there were 2,141 mites, belonging to 68 species of 49 families, for both nests, the adults were the best development phase, being the females the most abundant, with values of $85.57 \%$ for Nest I and $74.30 \%$ for Nest II. For the Nest I, the most abundant species was Macrocheles mexicanus (Evans \& Hyatt, 1963) (Macrochelidae), with a value of $50.64 \%$, being the females more abundant than the males. On the other hand, the most abundant species in Nest II was Eulohmannia sp. (13.61\%), registering females, deutonymphs and larvae. Diversity and richness were calculated for the two golden eagle nests, where Nest II presented a higher diversity and richness of species $\left(1-\mathrm{D}=0.927, D_{M g}=7.455\right)$. This work represents a first approach to the knowledge of mites associated with golden eagle nests.
\end{abstract}

Key words: Nests; mites; association; abundance; richness

\title{
INTRODUCCIÓN
}

Los nidos de las aves son un microhábitat óptimo para el establecimiento de una gran diversidad de artrópodos, ya sea de forma temporal o definitiva, la cual es reflejo de la composición, estructura y tamaño del nido, así como de las actividades del ave (Tryjanowski et al., 2001), ya que las condiciones del nido están sujetas a cambios cíclicos y dependen de la biología del ave (Bajerlein et al., 2006). Las interacciones que se desarrollan entre las aves y los artrópodos pueden llegar a establecerse a través del tiempo y tener un carácter co-evolutivo, y pueden asumir diversas formas (Bajerlein et al., 2006; Bloszyk et al., 2011). El componente más abundante de la artropodofauna asociada a nidos son los ácaros (Davidova \& Vasilev, 2012) los cuales, en su mayoría, comprenden a la fauna asociada con el microambiente del nido; este microambiente les confiere una alta dispersión y aislamiento, a excepción de los nidos de aves coloniales (Bajerlein et al., 2006).

El mayor número de registros de ácaros asociados a nidos de aves son en el orden Passeriformes (Krištofík et al., 1993; Krištofík et al., 2007; Bloszyk et al., 2009; Silva et al., 2018), comparado con los nidos de aves rapaces, en especial del orden Accipitriformes; a pesar de que estas pueden albergar una gran diversidad de invertebrados (Krištofík et al., 2009). Se han registrado 21 familias de ácaros asociadas con Accipitriformes que habitan sus plumas, cálamo, piel, tejido subcutáneo, tracto respiratorio y nidos (Philips, 2000). Según Philips y Dindal (1979), la artropodofauna de los nidos de aves rapaces puede dividirse en tres grupos: 1) la microfauna parásita, formada por los parásitos de las aves; 2) los animales saprófitos, que incluye a los invertebrados asociados con la descomposición de la carroña, excretas, egagrópilas y mudas, y 3) los organismos del humus, que incluye a aquellos invertebrados asociados con el material del nido como la madera y el suelo.

El águila real (Aquila chrysaetos canadensis Linnaeus, 1758) (Falconiformes: Accipitridae), se distribuye en Norteamérica, desde Alaska hasta el centro de México (Watson, 2010). Construye de uno a 14 nidos ubicados dentro de una superficie de 11 a $152 \mathrm{~km}^{2}$, área conocida como territorio de anidación 
(Kochert et al., 2002; Watson, 2010); estos nidos se localizan principalmente en paredes rocosas inaccesibles, pero también en árboles e inclusive en infraestructura humana (Kochert et al., 2002). El inicio de la temporada reproductiva, está definida con los primeros comportamientos de cortejo, los cuales consisten en la defensa del territorio, "vuelos nupciales" y la emisión de vocalizaciones; asimismo, y una vez seleccionado el sitio y/o nido, se inicia con la construcción y/o reparación del mismo con ramas y hojas frescas (Ferguson-Lees \& Christie, 2001; Kochert et al., 2002; Kochert \& Steenhof, 2012), la adición de material fresco al nido se mantiene después de la eclosión del polluelo, con el fin de mantener la higiene en el nido y repeler a los ectoparásitos (Wimberger, 1984; Heinrich, 2013). Por otra parte, la puesta consiste en uno a cuatro huevos, la incubación dura de 43 a 45 días y los pollos permanecen en el nido aproximadamente 10 semanas (Watson, 2010).

El objetivo del presente trabajo fue explorar la riqueza y diversidad de ácaros asociados a los nidos de águila real en México y aportar información sobre la asociación ácaros-nido.

\section{MATERIALES Y MÉTODOS}

Se recolectaron dos nidos de águila real el 19 de julio de 2013 por un mismo equipo de trabajo, y toda vez que se comprobó que en éstos no hubiera actividad de crianza. El Nido I se obtuvo de la localidad La Saladita, Sierra Fría, municipio de Genaro Codina, en el estado de Zacatecas. La Sierra Fría es una Región Prioritaria para la Conservación (RPC) dominada por bosques de encino, aunque también se presentan matorrales templados, áridos y subtropicales, chaparral, matorral (crasicaule, espinoso y rosetófilo) y pastizal natural (CONABIO, 2000). La hora de recolecta del Nido I fue entre las 9:00 y 12:00 horas. Por otra parte, el Nido II se recolectó en la Serranía de Juan Grande, municipio de El Llano, Aguascalientes (Fig. 1). El tipo de vegetación de esta serranía incluye pastizal-huizachal, matorral crasicaule y bosques de encino tipo arbustivo abierto (IMAE, 2006). La hora de recolecta de este nido fue entre las 19:00 y 20:30 horas.

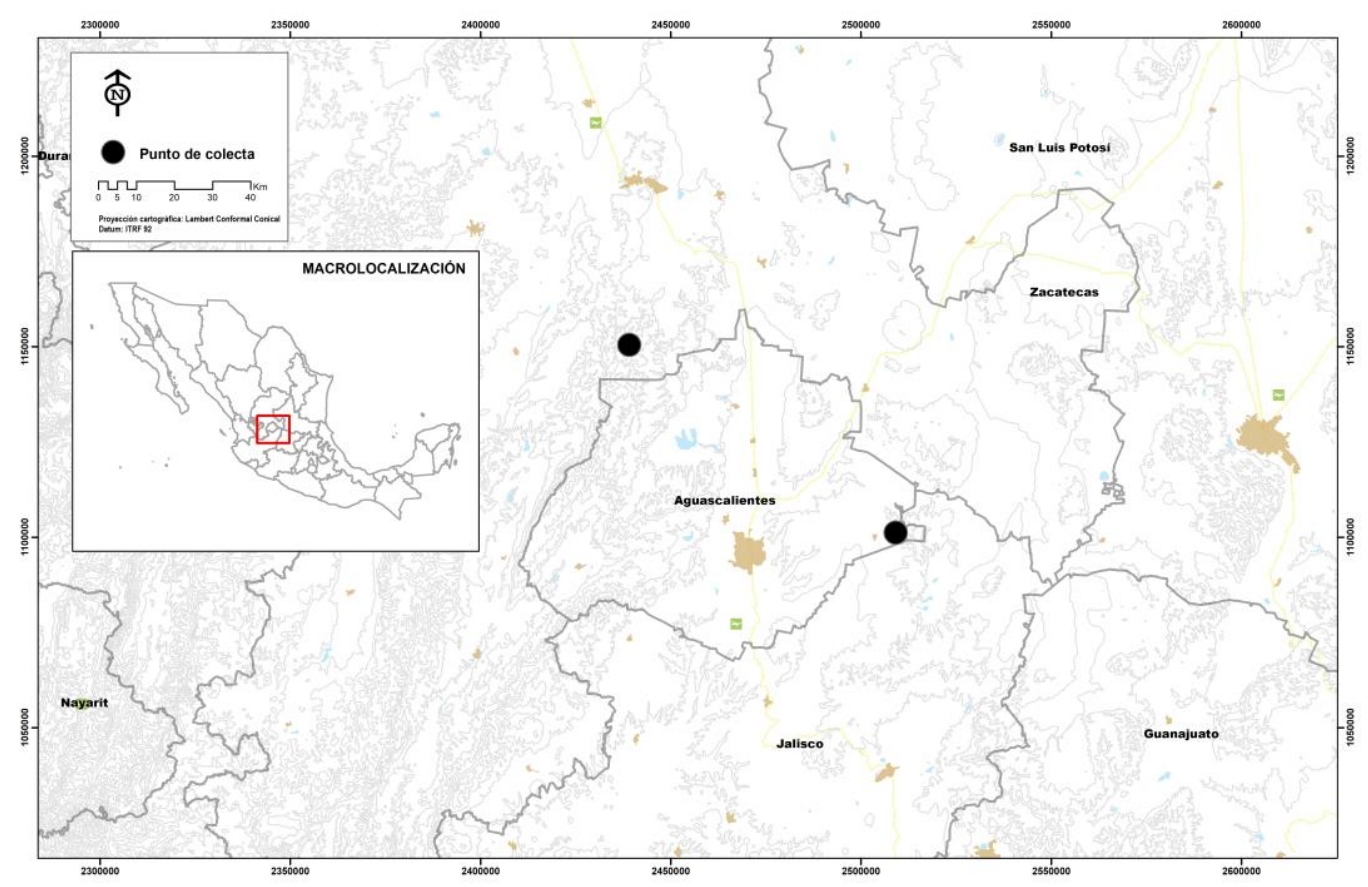

Figura 1. Mapa de ubicación de los sitios de recolecta de los nidos de águila real. 
Para ambas áreas de muestreo, se descendió con equipo de rapel, ya que los nidos se ubicaron en paredes rocosas (Figs. 4, 5); se desmontó la parte superficial de los nidos, lo cual correspondió a la región central y periférica de los nidos, aproximadamente $50 \mathrm{~cm}$ de área de los mismos, el material fue colocado en bolsas de plástico y en lugar fresco para su posterior procesamiento. Dos días después de la recolecta, el material fue procesado durante cinco días con la técnica de los embudos de Berlese-Tullgren con fuente de calor para acelerar el desecado, dicha técnica fue realizada en el laboratorio anexo al Laboratorio de Acarología "Dra. Isabel Bassols Batalla" de la Escuela Nacional de Ciencias Biológicas del Instituto Politécnico Nacional (ENCB-IPN); los organismos obtenidos fueron depositados en frascos de vidrio con alcohol etílico al 70\%, algunos de los organismos fueron aclarados con lactofenol durante 24 a 72 horas, para posteriormente elaborar laminillas semipermanentes con líquido de Hoyer, las que se llevaron a secado en una estufa a $45^{\circ} \mathrm{C}$ durante siete días. Se utilizó la clasificación de Lindquist et al. (2009a) para categorías superiores de la subclase Acari y la identificación de los ejemplares se realizó con el apoyo de guías especializadas de Balogh y Balogh (1992 a,b), Lindquist et al. (2009b), McDaniel (1979) y Walter et al. (2009). Los ácaros fueron depositados en la colección del Laboratorio de Acarología "Dra. Isabel Bassols Batalla", ENCB-IPN. La mayoría de los organismos se identificaron a nivel de género, debido al estadio de desarrollo encontrado, así como a la escaza literatura para la identificación a nivel de especie.

Se calculó el porcentaje de abundancia relativa (Ai\%) para ambos nidos, el cual está expresado como el número de individuos de la especie $i$ entre el número total de individuos de la muestra x 100 (modificado de Moreno, 2001); a partir de las abundancias obtenidas se calcularon rangos de abundancia para los dos nidos, obtenidos como la diferencia existente entre los valores máximo y mínimo observados y divididos entre cinco (no. de intervalos seleccionados) (Álvarez-González, s/a) (Cuadro 1).

Mediante el programa Past Versión 3.20 (Øyvind, 2018) se calculó la diversidad de especies a través de los índices de Simpson $\left(1-D\right.$; donde $D$, se obtiene como $=\left(\frac{1}{\Sigma(P i)^{2}}\right)$ y Margalef, $\left(D_{M g}=\frac{S-1}{\operatorname{lnN}}\right)$, respectivamente (Moreno et al., 2011).

\section{RESULTADOS}

Ambos nidos presentaron una constitución y estructura similares, variando en el tamaño: $100 \mathrm{~cm}$ de ancho x $150 \mathrm{~cm}$ de largo (NI) (Fig. 2) y $150 \mathrm{~cm}$ de ancho x $200 \mathrm{~cm}$ de largo (NII) (Fig. 3); constituidos principalmente por ramas de encino (Quercus sp.) y sotol (Dasylirion sp.); asimismo, presentaron restos de mamíferos de talla pequeña (Sylvilagus spp.), siendo el Nido I el que presentó mayor proporción de restos.

Para los dos nidos de águila real se encontraron 2,141 ácaros de 68 especies ubicados en 49 familias; en el Nido I se obtuvieron 312 ácaros de 26 especies de los cuales, nueve especies (ocho familias) pertenecen al orden Mesostigmata, cinco especies (cinco familias) al suborden Prostigmata, dos especies (dos familias) al cohorte Astigmatina y 10 especies de nueve familias pertenecientes al suborden Oribatida s.str. En cuanto al Nido II, se contabilizaron 1,829 ejemplares de 57 especies, de los cuales, 12 especies (nueve familias) pertenecen al orden Mesostigmata, 14 especies (10 familias) al suborden Prostigmata, cuatro especies dentro de tres familias al cohorte Astigmatina y 27 especies de 20 familias al suborden Oribatida s.str. Para ambos nidos, los adultos fueron la fase de desarrollo mejor representada, siendo las hembras las de mayor abundancia, con valores de $85.57 \%$ para el Nido I (267 $\circ$, $44 \hat{\gamma}$ y una

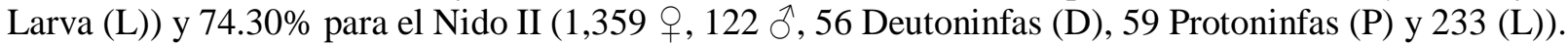

Para el Nido I, la especie más abundante fue Macrocheles mexicanus (Evans \& Hyatt, 1963) (Macrochelidae) (Fig. 2), con un valor del 50.64\%, siendo las hembras más abundantes que los machos 
(119 †, 39 ð), la segunda especie más abundante fue Iponemus truncatus (Ewing, 1939) (Tarsonemidae) con una abundancia del 33.01\%, donde se contabilizaron sólo hembras (103 + ) (Cuadro 1, 2).

Cuadro 1. Rangos de abundancia de ácaros para los nidos de águila real.

\begin{tabular}{ccc}
\hline RANGO DE ABUNDANCIA & NIDO I & NIDO II \\
\hline 1 & $40.561-50.621$ & $10.899-13.614$ \\
2 & $30.501-40.561$ & $8.188-10.899$ \\
3 & $20.441-30.501$ & $5.477-8.188$ \\
4 & $10.381-20.441$ & $2.766-5.477$ \\
5 & $0.321-10.381$ & $0.055-2.766$ \\
\hline
\end{tabular}

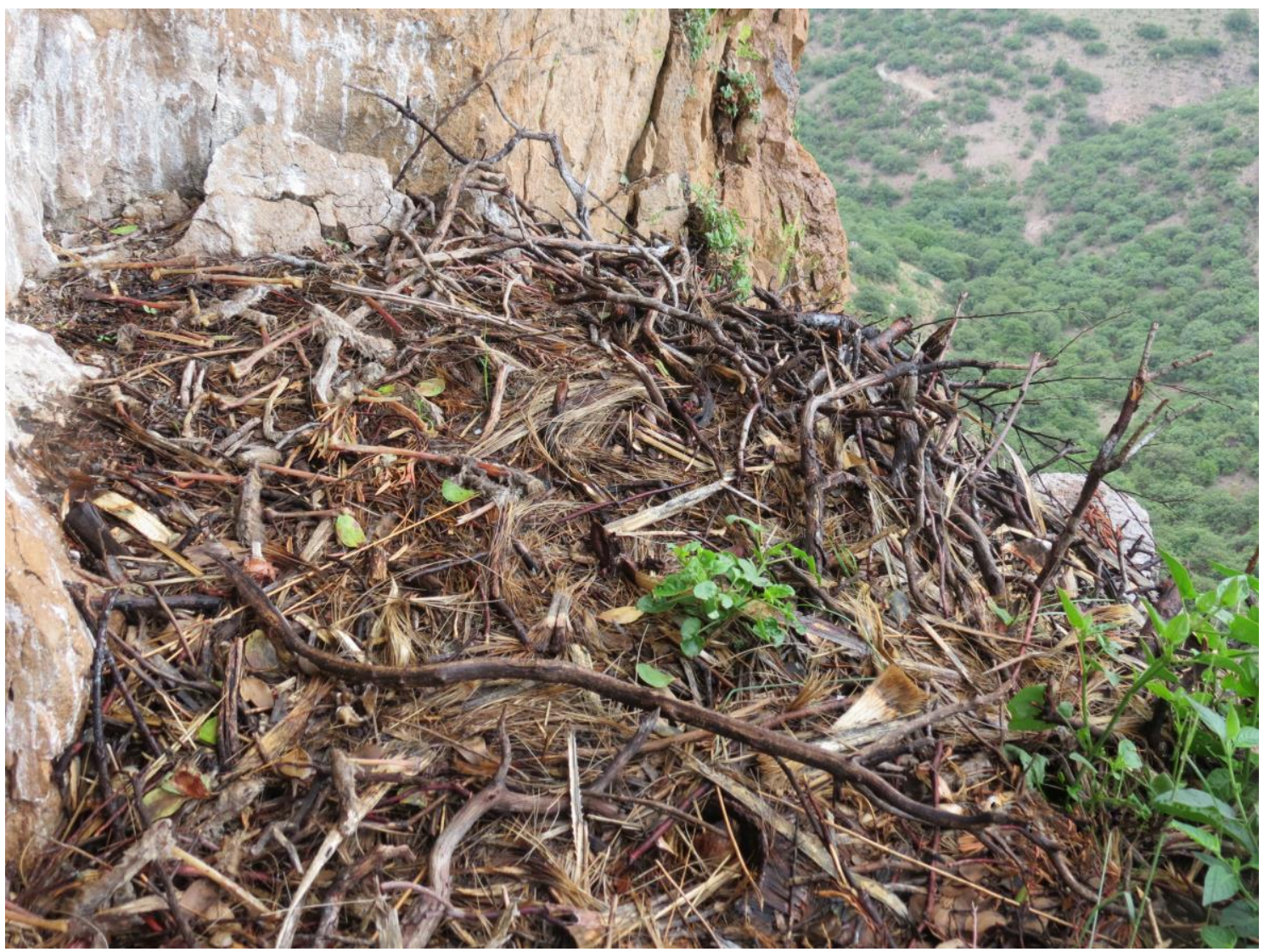

Figura 2. Vista del Nido I, localizado en la localidad La Saladita, Sierra Fría, municipio de Genaro Codina, Zacatecas.

En relación al Nido II, las especies más abundantes fueron Eulohmannia sp. (Eulohmanniidae) (13.61\%), I. truncatus (11.75\%), Arcoppia sp. (Oppiidae) (10.60\%) y M. mexicanus (10.55\%); donde Eulohmannia sp. presentó hembras, deutoninfas, protoninfas y larvas ( 8 , $40 \mathrm{D}, 30 \mathrm{P}, 171 \mathrm{~L})$, mientras que en I. truncatus y Arcoppia sp. sólo se observaron hembras con valores de 215 \& y 194 , respectivamente. En un segundo rango de abundancia, se encontraron únicamente adultos de $M$. mexicanus, siendo las hembras las más abundantes $(10.55 \% ; 132$ ᄋ , 61 §); en un tercer rango, Joshuella sp. (Gymnodamaeidae) presentó un porcentaje de abundancia del $5.96 \%$, constituido sólo por hembras $(109$ o) (Cuadro 1, 2). 
Damián-Chávez et al.: Ácaros asociados a nidos de águila real

Cuadro 2. Listado de las especies de ácaros identificadas en los nidos de águila real.

ㅇ- - Hembra; $\widehat{0}$ - Macho; D- Deutoninfa; P- Protoninfa; L- Larva; Ai\%-Porcentaje de abundancia relativa.

\begin{tabular}{|c|c|c|c|c|}
\hline TAXON & NIDO I & $\mathbf{A i} \%$ & NIDO II & $\mathbf{A i} \%$ \\
\hline \multicolumn{5}{|l|}{ SUPERORDEN PARASITIFORMES } \\
\hline \multicolumn{5}{|l|}{ ORDEN MESOSTIGMATA } \\
\hline \multicolumn{5}{|l|}{ Diplogyniidae } \\
\hline Cryptometasternum ca. natalense & - & - & $1 \delta^{\lambda}$ & 0.055 \\
\hline \multicolumn{5}{|l|}{ Thinozerconidae } \\
\hline $\begin{array}{l}\text { Thinozercon sp. } \\
\text { Urodinychidae }\end{array}$ & \multicolumn{3}{|c|}{ Urodinychidae } & - \\
\hline Uroobovella sp. & - & - & 1 ㅇ, $30^{\lambda}$ & 0.219 \\
\hline \multicolumn{5}{|l|}{ Zerconidae } \\
\hline Zercon farrieri & $1 \delta^{\lambda}$ & 0.321 & - & - \\
\hline Monozercon sp. & - & - & $2+, 6 \hat{0}$ & 0.437 \\
\hline \multicolumn{5}{|l|}{ Parasitidae } \\
\hline Vulgarogamasus sp. & - & - & $2 \hat{\jmath}$ & 0.109 \\
\hline Leptogamasus sp. & - & - & $2 \hat{\jmath}$ & 0.109 \\
\hline \multicolumn{5}{|l|}{ Veigaiidae } \\
\hline Gorirossia sp. & $10^{\lambda}$ & 0.321 & - & - \\
\hline \multicolumn{5}{|l|}{ Digamasellidae } \\
\hline Dendrolaelaps sp. & $1 \sigma^{\lambda}$ & 0.321 & 19 19, $27 \widehat{ठ}$ & 2.515 \\
\hline \multicolumn{5}{|l|}{ Rhodacaridae } \\
\hline \multirow{2}{*}{\multicolumn{5}{|c|}{ Eviphididae }} \\
\hline & & & & \\
\hline Alliphis ritcheri & - & - & 29 & 0.109 \\
\hline \multicolumn{5}{|l|}{ Macrochelidae } \\
\hline Macrocheles mexicanus & $119+, 39{ }^{\star}$ & 50.641 & $132+, 61{ }^{\top}$ & 10.552 \\
\hline \multicolumn{5}{|l|}{ Ascidae } \\
\hline Asca aphidioides & 1 우 & 0.321 & $4 ㅇ, 40^{\lambda}$ & 0.437 \\
\hline Melichares (=Blatisocius) dentriticus & - & - & 1 + $10^{\widehat{\lambda}}$ & 0.109 \\
\hline Melichares (=Blatisocius) pomorum & - & - & $3 \hat{\jmath}, 1 \mathrm{~L}$ & 0.219 \\
\hline \multicolumn{5}{|l|}{ Laelapidae } \\
\hline Haemogamasus occidentalis & $1 q$ & 0.321 & - & - \\
\hline Ololaelaps sp. & $1 q$ & 0.321 & - & - \\
\hline \multicolumn{5}{|l|}{ SUPERORDEN ACARIFORMES } \\
\hline \multicolumn{5}{|l|}{ ORDEN TROMBIDIFORMES } \\
\hline \multicolumn{5}{|l|}{ SUBORDEN PROSTIGMATA } \\
\hline \multicolumn{5}{|l|}{ Bdellidae } \\
\hline Bdella ca. ueckermanni & - & - & 6 우 & 0.328 \\
\hline Bdella longicornis & - & - & $15 \circ, 1 \mathrm{~L}$ & 0.875 \\
\hline Cyta spuria & - & - & 19 & 0.055 \\
\hline \multicolumn{5}{|l|}{ Cunaxidae } \\
\hline Cunaxa capreolus & - & - & $4 \mathrm{~L}$ & 0.219 \\
\hline Lupaeus minutus & - & - & $3 q$ & 0.164 \\
\hline \multicolumn{5}{|l|}{ Eupodidae } \\
\hline Protereunetes sp. & - & - & 1 우 & 0.055 \\
\hline \multicolumn{5}{|l|}{ Ereynetidae } \\
\hline Ereynetes sp. & $2 q$ & 0.641 & - & - \\
\hline \multicolumn{5}{|l|}{ Tydeidae } \\
\hline Coccotydaeulus sp. & $2 q$ & 0.641 & 2 우 & 0.109 \\
\hline Orthotydeus sp. & - & - & $15, 1 \mathrm{~L}$ & 0.875 \\
\hline Caeculidae & & & & \\
\hline Caeculus sp. & 19 & 0.321 & 5 오 & 0.273 \\
\hline
\end{tabular}




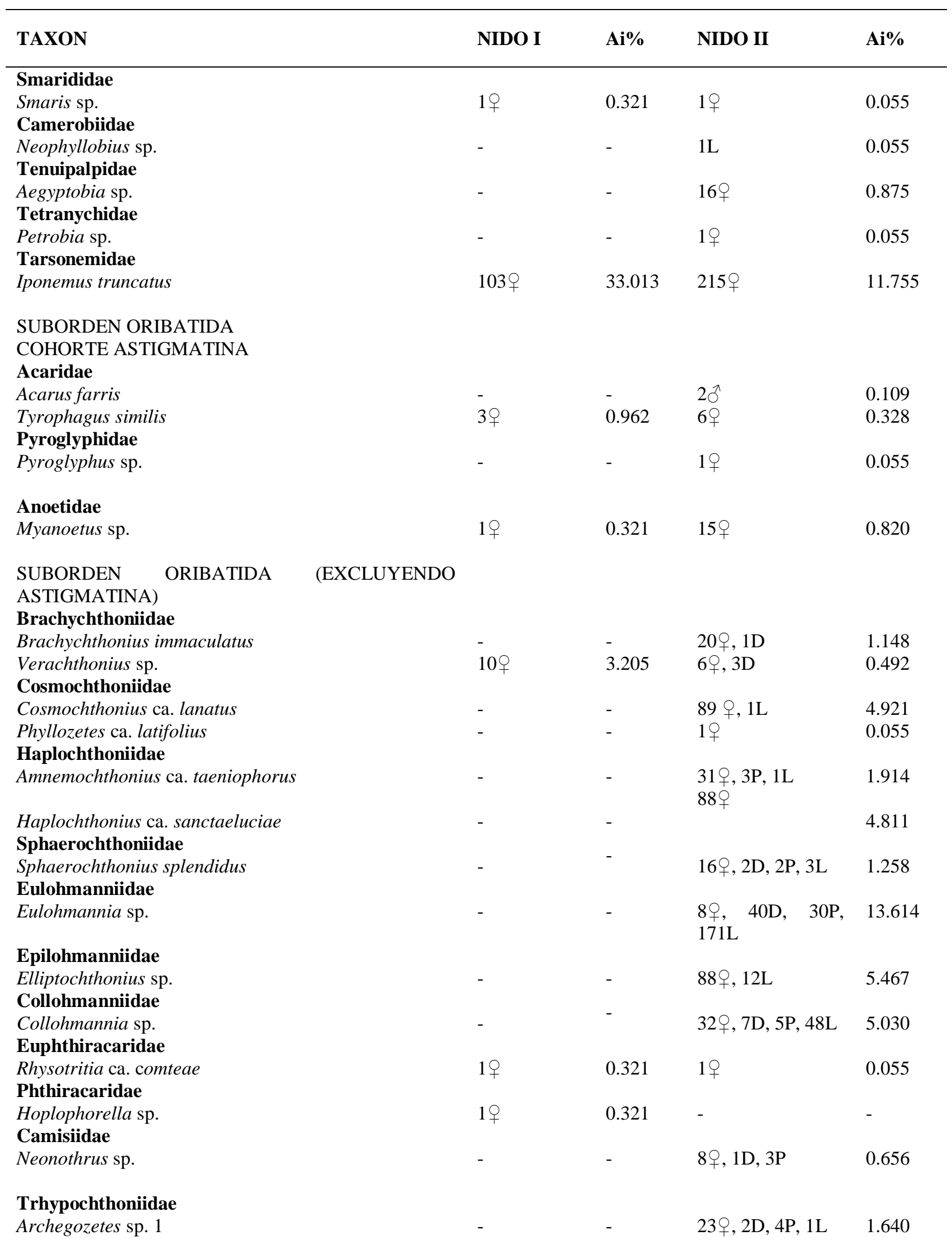




\begin{tabular}{|c|c|c|c|c|}
\hline TAXON & NIDO I & $\mathbf{A i} \%$ & NIDO II & $\mathbf{A i} \%$ \\
\hline $\begin{array}{l}\text { Archegozetes sp. } 2 \\
\text { Gymnodamaeidae }\end{array}$ & - & - & $3 q$ & 0.164 \\
\hline $\begin{array}{l}\text { Joshuella sp. } \\
\text { Damaeidae }\end{array}$ & - & - & $109+$ & 5.960 \\
\hline Allobelba sp. & 8 우 & 2.564 & - & - \\
\hline \multicolumn{5}{|l|}{ Oppiidae } \\
\hline Vietoppia sp. & - & - & 25 웅 & 1.367 \\
\hline Pseudoamerioppia sp. & 1 우 & 0.321 & - & - \\
\hline $\begin{array}{l}\text { Arcoppia sp. } \\
\text { Tectocepheidae }\end{array}$ & - & - & 194 웅 & 10.607 \\
\hline $\begin{array}{l}\text { Tectocepheus velatus } \\
\text { Cymbaeremaeidae }\end{array}$ & - & - & $2 q$ & 0.109 \\
\hline $\begin{array}{l}\text { Scapheremaeus mahunkaius } \\
\text { Micreremidae }\end{array}$ & - & - & 1 우 & 0.055 \\
\hline $\begin{array}{l}\text { Micreremus sp. } \\
\text { Passalozetidae }\end{array}$ & $1 \mathrm{D}$ & 0.321 & - & - \\
\hline $\begin{array}{l}\text { Passalozetes sp. } \\
\text { Haplozetidae }\end{array}$ & $1 q$ & 0.321 & 10 우 & 0.547 \\
\hline Mancoribates sp. & - & - & 62 우 & 3.390 \\
\hline $\begin{array}{l}\text { Rostrozetes sp. } \\
\text { Oribatulidae }\end{array}$ & $1 q$ & 0.321 & $1 q$ & 0.055 \\
\hline $\begin{array}{l}\text { Lucoppia felderi } \\
\text { Oripodidae }\end{array}$ & - & - & 42 ㅇ, $3 \hat{0}$ & 2.460 \\
\hline $\begin{array}{l}\text { Oripoda sp. } \\
\text { Zetomotrichidae }\end{array}$ & - & - & 1 운 & 0.055 \\
\hline $\begin{array}{l}\text { Zetomotrichidae bidentata } \\
\text { Galumnidae }\end{array}$ & - & - & 1 우 & 0.055 \\
\hline Pilogalumna sp.1 & - & - & $14 ㅇ$ & 0.765 \\
\hline Pilogalumna sp. 2 & 5 우 & 1.603 & - & - \\
\hline Galumna unica & 40 & 1.282 & $18 ㅇ$ & 0.984 \\
\hline
\end{tabular}

Diversidad y riqueza de especies en los dos nidos de águila real. La diversidad de los dos nidos de águila real mediante el índice de Simpson (1-D) fue de 0.632 para el Nido I y de 0.927 para el Nido II; para el índice de Margalef $\left(D_{M g}\right)$, fue de 4.353 para el Nido I y 7.455 para el Nido II, ambos índices en conjunto infieren que los dos nidos presentan valores de diversidad altos, debido a que presentan una distribución de abundancias más equitativa entre las especies; por otra parte, el Nido II es el que presenta una mayor diversidad de especies, ya que registró un total de 57 especies en comparación a las 26 del Nido I (Cuadro $3)$.

Cuadro 3. Índices de abundancia, riqueza de especies y diversidad de ácaros colectados en los dos nidos de águila real.

\begin{tabular}{lll}
\hline ÍNDICE & NIDO I & NIDO II \\
\hline Número total de individuos $(\mathrm{N})$ & 312 & 1,829 \\
Número total de especies (S) & 26 & 57 \\
Simpson $(1-D)$ & 0.632 & 0.927 \\
Margalef $\left(D_{M g}\right)$ & 4.353 & 7.455 \\
\hline
\end{tabular}




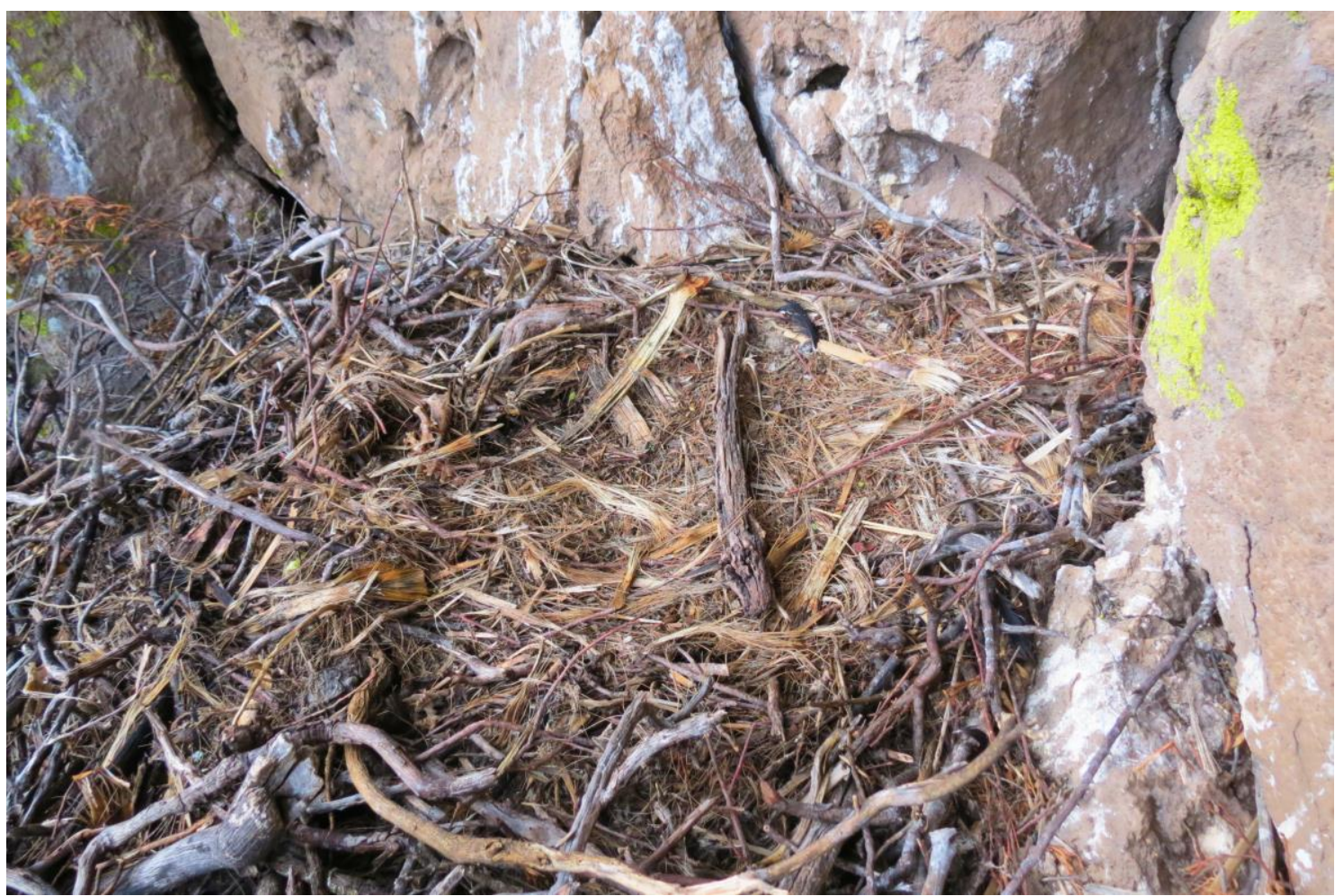

Figura 3. Vista del Nido II, recolectado en la Serranía de Juan Grande, municipio de El Llano, Aguascalientes.

\section{DISCUSIÓN}

La presencia y abundancia de ácaros en los nidos de águila real está determinada por diferentes factores, entre los que destacan: las actividades del ave previas al vuelo del juvenil, la composición y tamaño del nido y el tiempo de abandono por parte del juvenil con respecto al día de recolecta. Las rapaces, incluyendo el águila real, adicionan material fresco de vegetación a los nidos para disminuir la cantidad de ectoparásitos; sin embargo, esta actividad es realizada pocos días después de la eclosión del polluelo (Wimberger, 1984; Heinrich, 2013); después de este evento, las actividades principales de los padres se basan, en el cuidado de las crías, el suministro de alimento y en la protección del área cercana al nido (Watson, 2010), por lo que, al garantizar el éxito reproductivo del polluelo, los padres dejan de adicionar material vegetal fresco al nido, lo cual crea la oportunidad de establecimiento y colonización de ácaros sobre estas estructuras. En relación al tamaño de los nidos, entre más grande sea la estructura, mayor cantidad de ácaros pueden albergar (Tryjanowski et al., 2001); por otro lado, el abandono de los nidos por parte del juvenil lleva consigo la ausencia en el suministro de alimento para la acarofauna y demás artrópodos nidícolas, este abandono también implica la degradación paulatina de la estructura del nido (composición y forma), por lo que ambos sucesos resultan en una disminución de las poblaciones residentes de los nidos, al ser éste un microhábitat dependiente del águila real (Bajerlein et al., 2006).

En referencia al abandono de los nidos en 2013, éste aconteció en diferentes tiempos: el abandono del Nido I ocurrió a finales de mayo, mientras que el abandono del Nido II sucedió a mediados de junio, por lo que, con aproximadamente un mes y medio de abandono del Nido I con respecto a la fecha de recolecta, y al presentar un menor tamaño en comparación al Nido II, el Nido I presentó una menor abundancia y diversidad de especies con tan sólo 312 organismos contabilizados, 26 especies determinadas y un índice de riqueza del 4.353 , contrario a lo obtenido en el Nido II $(\mathrm{N}=1,829 ; \mathrm{S}=57$; $\left.D_{M g}=7.455\right)$. 


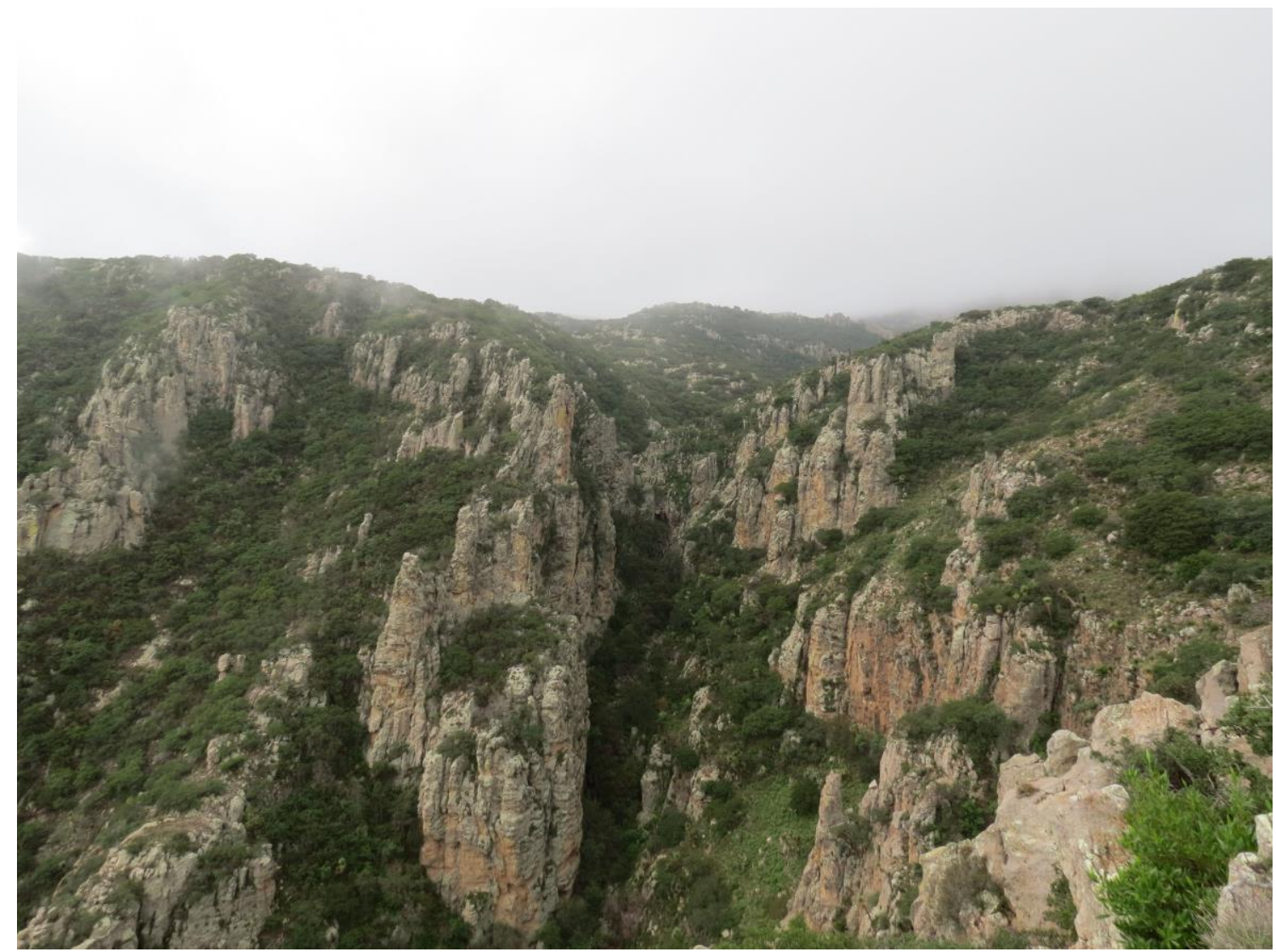

Figura 4. Vista panorámica de la Sierra Fría, Zacatecas, territorio de anidación del águila real.

En relación al orden Mesostigmata, se observó que las hembras de M. mexicanus fueron más abundantes respecto a los machos (NI: 119 ㅇ, 39 ภ; NII: 132 ㅇ, 61 §), debido a que éstas son más longevas, se ha documento que en varios arácnidos los machos mueren después de que se reproducen, mientras que las hembras se mantienen vivas durante un periodo de tiempo mayor, por lo que la proporción de sexos estará orientada hacia las hembras (Bruce \& Wrensch, 1990; Beccaloni, 2009). La mayoría de las hembras de ambos nidos se encontraron en estado grávido y se observó que eran huéspedes de las hembras de I. truncatus, las cuales se encontraron adheridas a la placa dorsal del macroquélido hembra, generalmente en la región posterior de esta placa. Se conoce que las hembras de I. truncatus son parásitas de escarabajos descortezadores (Lindquist, 1969); por lo que, para el presente estudio, estas podrían considerarse parásitas o bien, puede estarse llevando a cabo una relación forética entre ambas especies, donde las hembras de M. mexicanus favorecen la dispersión del prostigmado dentro del nido, puesto que estas presentan un tamaño mayor respecto al prostigmado ( + M. mexicanus: $\mathrm{L}=723 \mu \mathrm{m} \times \mathrm{A}=$ $285 \mu \mathrm{m} ;$; I. truncatus: $\mathrm{L}=165 \mu \mathrm{m} \times \mathrm{A}=88 \mu \mathrm{m})$.

El suborden Prostigmata estuvo representado por cinco especies en el Nido I y 14 especies en el Nido II, donde I. truncatus fue la especie más abundante de este suborden con valores de $33.01 \%$ (NI) y $11.75 \%$ (NII), esta abundancia de individuos se asume a la acumulación del material orgánico presente en los nidos, la cual favorece la disponibilidad de alimento, así como la presencia de otras especies y que con ello favorece la dispersión de I. truncatus. Las especies de este suborden son comunes en hojarasca, musgo, liquen y en hábitats similares; asimismo, la mayoría de las especies determinadas para este estudio, son de hábitos depredadores (Bdella ca. ueckermanni, Cunaxa capreolus, Caeculus sp., Neophyllobius sp.) y fitófagos (Aegyptobia sp., Petrobia sp., Cunaxa capreolus) (De León, 1958; Zaher et al., 1975; Otto, 1993; Walter et al., 2009; Vieira de Souza, 2010; Hernandes et al., 2011; Muhammad \& 
Muhammad, 2011; Khanjani et al., 2013; Skvarla et al., 2014), a excepción de Orthotydeus sp., quien es un organismo saprófago y micetófago, el cual obtiene su alimento de la materia orgánica en descomposición presente en el Nido II (Pérez-Otero \& Mansilla-Vázquez, 1997).

El cohorte Astigmatina fue el grupo menos representativo de los dos nidos de águila real, con tan sólo dos especies para el Nido I y cuatro para el Nido II. Para el Nido I, la especie más abundante fue Tyrophagus similis (Volgin, 1949) con tres hembras contabilizadas. Esta especie ha sido recolectada en pajareras de Ara ararauna (Linnaeus, 1758) (guacamayo azul) y Nymphicus hollandicus (Kerr, 1792) (cacatúa ninfa) siendo una especie dominante (Solarz et al., 2004); esta referencia nos indica, que la especie no encuentra las condiciones óptimas para su establecimiento es lugares con abundancia de materia orgánica. Por otra parte, Myanoetus sp. fue el más abundante en el Nido II con 15 hembras, se conoce que este género es común sobre insectos y otros artrópodos (McDaniel, 1979), y que al encontrase estos organismos en el nido (hormigas, psudoescopiones y coleópteros), se asume a que contribuyen a la presencia de este género en el nido.

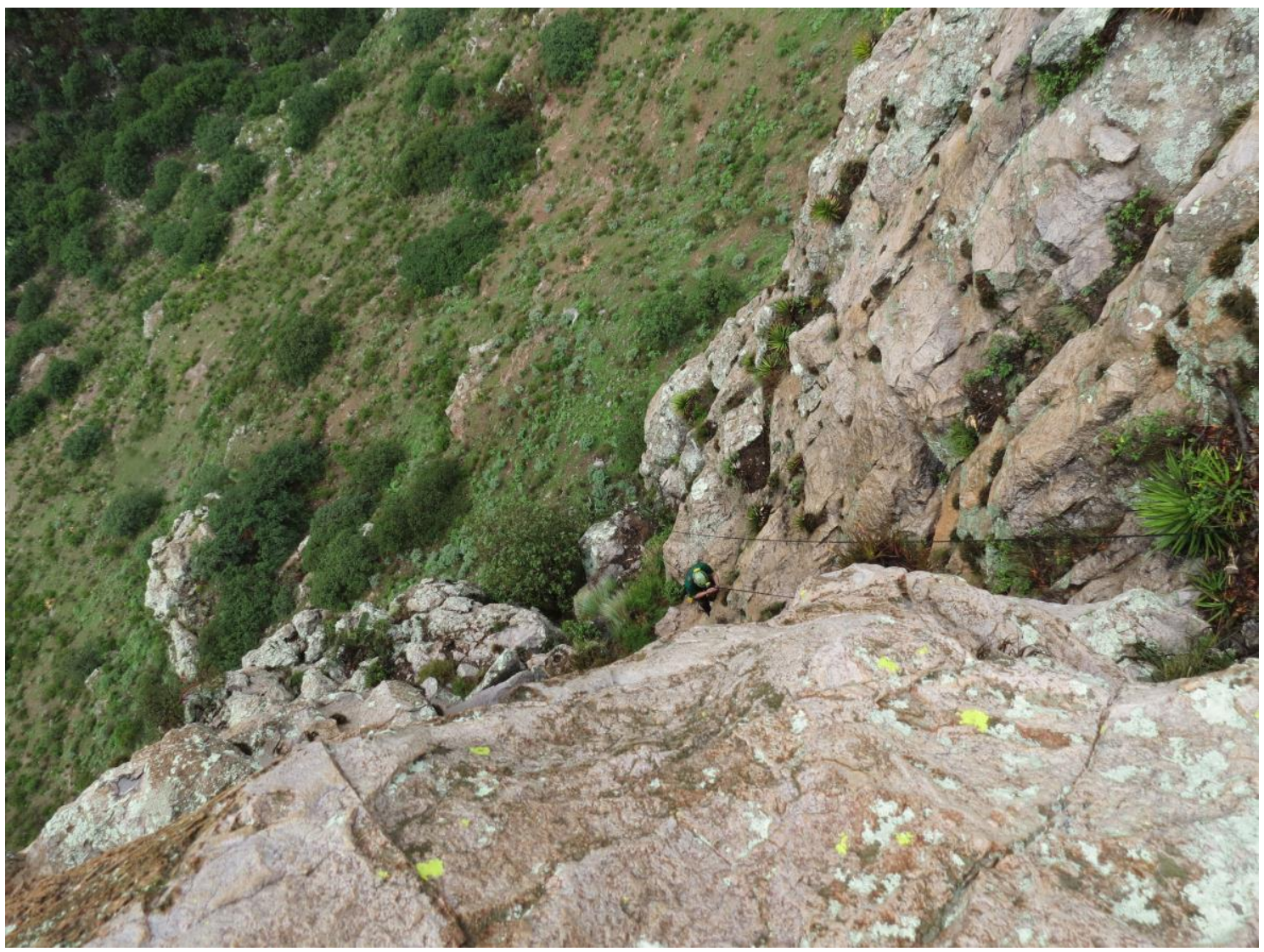

Figura 5. Descenso al Nido I para la recolecta del material.

Los ácaros oribátidos son uno de los grupos mejor representados en cuanto al número de especies y abundancias (Urhan et al., 2008), y para este estudio, no fueron la excepción, ya que se determinaron diez especies para el Nido I (33 ejemplares) y 27 para el Nido II (1,237 ejemplares). Su abundancia en comparación con las demás categorías superiores de la Subclase Acari se asume a que el suelo de los nidos presenta una alta porosidad, así como grandes cantidades de materia orgánica (Andrén \& Lagerlöf, 1983; Pertersen \& Luxton, 1982) que favorecen la abundancia y diversidad de estos organismos. Para el Nido I, la especie más abundante fue Brachychthonius immaculatus (Forsslund, 1942) con diez hembras, esta especie se ha encontrado asociada a liquen, musgo y suelos (Schatz, 1995); por otra parte, Allobelba 
sp., fue el segundo organismo más abundante dentro del nido y generalmente se le encuentra asociado al detritos orgánico (McDaniel, 1979); de este género se contabilizaron ocho hembras grávidas con un aproximado de cinco a seis huevos en su interior, siendo registrada nuevamente esta proporción orientada a las hembras, otra especie que también presentó esta proporción dentro del Nido I fue Rhysotritia ca. comteae con la característica de presentar tres huevos sumamente ornamentados en su interior. Por otro lado, las restantes especies determinadas en este nido fueron Hoplophorella sp., Pseudoamerioppia sp., Micreremus sp., Pilogalumna sp. 2 y Rostrozetes sp. (Franklin et al., 2004; Seniczak \& Seniczak, 2006; Bernal-Rojas et al., 2009; Vázquez-González, 2012; Weigmann, 2013) las cuales se distribuyen en suelos con alta humedad, mientras que Passalozetes sp. se registra en ambientes áridos y en suelos de altitudes altas (Martínez \& Herrero, 2006), tal como los nidos de águila real estudiados. En relación con el Nido II, destaca el género Eulohmannia sp. quien presentó hembras, deutoninfas, protoninfas y larvas, lo que sugiere que la longevidad de los machos es menor a la de las hembras; a su vez, los resultados obtenidos nos señalan la baja abundancia de hembras dentro de este género sugiriendo el término de su ciclo de vida dentro de este microhábitat, observándose una alta abundancia de estadios inmaduros $(8 \%, 40 \mathrm{D}, 30 \mathrm{P}$, 171 L). Del mismo modo, Collohmannia sp. (32 o, 7 D, 5 P, 48 L), Archegozetes sp. 1 (23 9,2 D, 4 P, 1 L) y Sphaerochthonius splendidus (16 +2 D, 2 P, 3 L) presentan el mismo patrón de estadios de desarrollo que Eulohmannia sp. (excepto machos y tritoninfas); sin embargo, este patrón se da en diferentes proporciones, siendo las hembras más abundantes que sus respectivos estados inmaduros. En relación a las demás especies dentro de este nido, la gran mayoría de ellas se distribuyen en ambientes húmedos; sin embargo, también se presentan especies con preferencias por ambientes semiáridos y áridos tales como Joshuella sp., Elliptochthonius sp. y Arcoppia sp. (Shepherd et al., 2002; Arroyo \& Iturrondobeitia, 2003; Ermilov et al., 2012; Bernal-Rojas et al., 2009).

Al comparar el presente trabajo con estudios de nidos de otras aves rapaces, se observa que se comparten algunos patrones para el orden Mesostigmata, ya que los estudios publicados se basan sólo en este grupo de ácaros. Gwiazdowicz et al. (2006) analizaron 34 nidos de Haliaeetus albicilla (Linnaeus, 1758), conocida como pigargo europeo o águila marina, donde determinaron 86 especies de mesostigmados, mientras que en el presente trabajo se identificaron cinco especies para el Nido I y 14 para el Nido II, no obstante se comparten algunas especies o géneros: Alliphis sp. (Eviphididae), Asca aphidioides (Ascidae), Dendrolaelaps sp., Haemogamasus sp. (Laelapidae), Macrocheles sp., Uroobovella sp. (Urodinychidae), Vulgarogamasus sp. (Parasitidae) y Zercon sp. (Zerconidae). Dendrolaelaps y Macrocheles que, aunque se presentan en el presente estudio, son considerados géneros comunes y diversos en cualquier estudio de nidos de aves (Bloszyk et al., 2005; Gwiazdowicz et al., 2006; Bloszyk et al., 2009; Bloszyk et al., 2011; Davidova \& Vasilev, 2012).

Dado que sólo fueron dos nidos de águila real recolectados, la muestra puede ser no significativa; por lo que se sugiere incrementar los estudios sobre los nidos de esta rapaz. Para incrementar el conocimiento en la relación águila real-nido-ácaro, se necesita conocer el ciclo reproductivo de la pareja de águila real objetivo; es decir, conocer la fecha de inicio de la construcción o renovación del nido; determinación taxonómica del material florístico usado para la construcción; éxito reproductivo de la pareja; fecha de abandono de los nidos por parte del juvenil o juveniles así como la fecha de recolecta del nido, esta última, deberá garantizar la no perturbación de la especie. Con estos parámetros, se contribuirá a cubrir los espacios vacios de información, y se incrementará el conocimiento de la dinámica de los nidos.

Este es el primer trabajo de ácaros asociados a nidos de águila real para México, donde la presencia de ácaros está íntimamente ligada a las actividades del ave, y a la estructura y composición de los nidos, tal y como se pudo observar en el Nido II, el cual presento un mayor número de individuos y una mayor diversidad de ácaros en comparación al Nido I. 
Agradecimientos. A Víctor Manuel Córdova Tabares, Francisco Alejandro Rodríguez Salas y David "Tarzán" Carreón Araiza, por su ayuda en la recolecta de los nidos, por su colaboración en el trabajo de campo a Rossana Bernal Martínez y al Sr. José Esquivel. A Margarita Ojeda e Isabel Sánchez Rocha ${ }^{\dagger}$ por su ayuda en la identificación de los ácaros oribátidos. A Ricardo Paredes-León por la revisión y sus comentarios que enriquecieron al manuscrito. Al proyecto SIP 20141374 de la ENCB-IPN. A dos revisores anónimos que con sus comentarios enriquecieron al manuscrito.

\section{LITERATURA CITADA}

Álvarez-González, F. (s/a) Estadística descriptiva. Available at: http://www.uco.es/zootecniaygestion/img/pictorex/27_12_49_7.pdf (acceso en diciembre de 2018).

Andrén, O., Lagerlöf, J. (1983) Soil fauna (Microarthropods, enchytraeids, nematodes) in Swedish agricultural cropping systems. Acta Agriculturae Scandinavica, 33, 33-52. https://doi.org/10.1080/00015128309435350

Arroyo, J., Iturrondobeitia, J. C. (2003) Listado sistemático de los oribátidos (Arachnida, Acari, Oribatida) de diferentes medios edáficos de la Provincia de Burgos, España. Revista Ibérica de Aracnología, 8, 103-110.

Bajerlein, D., Loszyk, J. B., Gwiazdowicz, D., Ptaszyk, J., Halliday, B. (2006) Community structure and dispersal of mites (Acari, Mesostigmata) in nests of the white stork (Ciconia ciconia). Biologia, 61, 525-530. https://doi.org/10.2478/s11756-006-0086-9

Balogh, J., Balogh, P. (1992a) The oribatid mites genera of the World. Vol. I. The Hungarian National Museum Press. Hungary, 263 pp.

Balogh, J., Balogh, P. (1992b) The oribatid mites genera of the World. Vol. II. The Hungarian National Museum Press. Hungary, 373 pp.

Beccaloni, J. (2009) Arachnids. Natural History Museum. Londres, Reino Unido, 320 pp.

Bernal-Rojas, A., Cataño-Meneses, G., Palacios-Vargas, J. G., García-Calderón, N. E. (2009) Oribatid mites and springtails from a coffee plantation in Sierra Sur, Oaxaca, Mexico. Revista Pesquisa Agropecuária Brasileira, 44, 988-995. http://dx.doi.org/10.1590/S0100-204X2009000800030

Bloszyk, J., Drazina, T., Gwiazdowicz, D. J., Halliday, B., Goldyn, B., Napierala, A., Rybska, E. (2011) Mesostigmatic mites (Acari: Mesostigmata) in nests of the Eurasian griffon vulture (Gyps fulvus) in Croatia. Biologia, 66, 335-339. http://dx.doi.org/10.2478/s11756-011-0011-8

Bloszyk, J., Gwiazdowicz, D. J., Bajerlein, D., Halliday, R. B. (2005) Nests of the white stork Ciconia ciconia (L.) as a habitat for mesostigmatic mites (Acari, Mesostigmata). Acta Parasitologica, 50, 171-175.

Bloszyk, J., Gwiazdowicz, D. J., Halliday, B., Dolata, P. T., Goldyn, B. (2009) Nests of the black stork Ciconia nigra as a habitat for mesostigmatid mites (Acari: Mesostigmata). Biologia, 64, 962-968. http://dx.doi.org/10.2478/s11756-009-0146-z

Bruce, W. A., Wrensch, D. (1990) Reproductive potential, sex ratio, and mating efficiency of the straw itch mite (Acari: Pyemotidae). Journal of Economic Entomology, 83, 384-391. http://dx.doi.org/10.1093/jee/83.2.384

CONABIO (2000) Regiones Terrestres Prioritarias de México: Sierra Fría, RTP-66. Available at: http://www.conabio.gob.mx/conocimiento/regionalizacion/doctos/rtp_066.pdf (acceso en julio 2017).

Davidova, R., Vasilev, V. (2012) Community structure of mesostigmatic mites (Acari: Parasitiformes) in nests of the great tit (Parus major). Folia Faunistica Slovaca, 17, 191-196. 
De León, D. (1958) The genus Neophyllobius in Mexico (Acarina: Neophyllobiidae). The Florida Entomologist, 41, 173-181.

Ermilov, S. G., Niedbala, W., Anichkin, A. E. (2012) Oribatid mites of Dong Nai Biosphere Reserve (Cat Tien National Park) of Southern Vietnam, with description of a New Species of Pergalumna (Acari, Oribatida, Galumnidae). Acarina, 20, 20-28.

Ferguson-Lees, J., Christie, D. A. (2001) Raptors of the World. Houghton Mifflin. Boston, 992 pp.

Franklin, E., Hayek, T., Fagundes, E. P., Silva, L. L. (2004) Oribatid mite (Acari: Oribatida) contribution to decomposition dynamic of leaf litter in primary forest, second growth, and polyculture in the Central Amazon. Brazilian Journal of Biology, 64, 59-72.

Gwiazdowicz, D. J., Bloszyk, J., Bajerlein, D., Halliday, R. B., Mizera, T. (2006) Mites (Acari: Mesostigmata) inhabiting nests of the white-tailed sea eagle Haliaeetus albicilla (L.) in Poland. Entomologica Fennica, 17, 366-372.

Heinrich, B. (2013) Why does a hawk build with Green nesting material? Northeastern Naturalist, 20, 209-218. https://doi.org/10.1656/045.020.0202

Hernandes, F. A., De O Bernardi, L. F., Ferreira, R. L. (2011) Snout mites from caves in Brazil, with descriptions of a new species (Acari: Trombidiformes: Bdellidae). Journal of Natural History, 45, 799-812.

https://doi.org/10.1080/00222933.2010.535919

IMAE (2006) Estudio Técnico Justificativo: Área de protección del águila real (Aquila chrysaetos) de la Serranía de Juan Grande en el Ejido Palo Alto, municipio de El Llano, Aguascalientes, México. Available at:

https://www.researchgate.net/profile/Luis_Lozano_Roman/publication/284899837_Estudio_tecni co_justificativo_para_la_declaratoria_del_Cerro_de_Juan_Grande_como_area_de_proteccion_del _aguila_real/links/5659f58e08aefe619b231578/Estudio-tecnico-justificativo-para-la-declaratoriadel-Cerro-de-Juan-Grande-como-area-de-proteccion-del-aguila-real.pdf (acceso julio de 2017).

Khanjani, M., Zahiri, B., Khanjani, M. (2013) A new species of Aegyptobia (Acari: Tenuipalpidae) from Hamedan province, Iran. Persian Journal of Acarology, 1, 49-55. http://dx.doi.org/10.22073/pja.v2i1.9948

Kochert, M. N., Steenhof, K. (2012). Frequency of nest use by golden eagle in southwestern Idaho. Journal of Raptor Research, 46, 248-257. https://doi.org/10.3356/JRR-12-00001.1

Kochert, M. N., Steenhof, K., McIntyre, C. L., Craig, E.H. (2002) Golden Eagle (Aquila chrysaetos). Pp. 1-44. In: Poole, A., Gill, F. (Eds.). The Birds of North America. The Birds of North America Inc., Philadelphia.

Krištofík, J., Mašán, P., Šustek, Z. (2007) Arthropods (Pseudoscorpionidea, Acarina, Coleoptera, Siphonaptera) in nests of the bearded tit (Panurus biarmicus). Biologia, 62, 749-755. http://dx.doi.org/10.2478/s11756-007-0142-0

Krištofík, J., Mašán, P., Šustek, Z., Gajdos, P. (1993) Arthropods in the nests of penduline tit (Remiz pendulinus). Biologia, 48, 493-505.

Krištofík, J., Mašán, P., Šustek, Z., Karaska, D. (2009) Arthropods in the nests of lesser spotted eagle (Aquila pomarina). Biologia, 64, 974-980. http://dx.doi.org/10.2478/s11756-009-0148-x

Lindquist, E. E., Krantz, G. W., Walter, D. E. (2009a) Chapter eight: Classification. Pp. 97-103. In: Krantz, G. W., Walter, D. E. (Eds.). A manual of Acarology. Third Edition, Tech University Press, Texas.

Lindquist, E. E., Krantz, G. W., Walter, D. E. (2009b) Chapter twelve: Order Mesostigmata. Pp. 124232. In: Krantz, G. W., Walter, D. E. (Eds.). A manual of Acarology. Third Edition, Tech University Press, Texas.

Lindquist, E. E. (1969) Review of Holarctic tarsonemid mites (Acarina: Prostigmata) parasitizing eggs of Ipine bark beetles. The Memoirs of the Entomological Society of Canada, 60, 1-111. 
https://doi.org/10.4039/entm10160fv

Martínez, P. A., Herrero, A. C. (2006) A new species of Passalozetes (Acari: Oribatida) from the Andes mountains of Argentina. Zootaxa, 1160, 49-55.

McDaniel, B. (1979) How to know the mites and ticks. The pictures Key Nature Series. United States of America, $335 \mathrm{pp}$.

Moreno, C. E. (2001) Métodos para medir la biodiversidad. Vol. 1. M\&T-Manuales y Tesis SEA. España, $84 \mathrm{pp}$.

Moreno, C. E., Barragán, F., Pineda E., Pavón, N. P. (2011) Reanálisis de la diversidad alfa: alternativas para interpretar y comparar información sobre comunidades ecológicas. Revista Mexicana de Biodiversidad, 82, 1249-1261.

Muhammad, A. S., Muhammad, A. (2011) Description and distribution of a new species, Petrobia chaudhrii (Acarina: Tetranychidae) from Punjab, Pakistan. Pakistan Journal of Life Social Sciences, 9, 28-32.

Otto, J. C. (1993) A new species of Microcaeculus from Australia (Acarina: Caeculidae), with notes on its biology and behavior. International Journal of Acarology, 19, 3-13. https://doi.org/10.1080/01647959308683533

Øyvind, H. (2018) PAST, Reference manual. Natural History Museum. University of Oslo, Noruega, 264 pp.

Pérez-Otero, R. \& Mansilla-Vázquez, P. (1997) Acarofauna asociada a Pinus spp. en Galicia. Actas de I Congreso Forestal Hispano Luso y II Congreso Forestal Español. España, 349-353 pp.

Petersen, H., Luxton, M. (1982) A comparative analysis of soil fauna populations and their role in decomposition processes. Oiko, 39, 288-388. https://doi.org/10.2307/3544689

Philips, J. R., Dindal, D. L. (1979) The acarine community of nests of birds of prey. Pp. 559-562. In: Rodriguez, J. G. (Ed.). Recent advances in Acarology. Academic Press, United State of America.

Philips, J. R. (2000) A review and checklist of the parasitic mites (Acarina) of the Falconiformes and Strigiformes. Journal of Raptor Research, 34, 210-231.

Schatz, H. (1995) Hornmilben in Trockenrasenböden des Virgentales (Osttirol, Österreich). 2. Teil: Faunistik (Acari: Oribatida). Berichte des Naturwissenschaftlichen-MedizinischenVerein Innsbruck, 82, 121-144.

Seniczak, S., Seniczak, A. (2006) Oribatid mites (Acari) of some habitats on Rhodes Island (Greece). Biological Letters, 43, 215-219.

Shepherd, U. L., Brantley, S. L., Tarleton, C. A. (2002) Species richness and abundance patterns of microarthropods on cryptobiotic crusts in a piñon-juniper habitat: a call for greater knowledge. Journal of Arid Environments, 52, 349-360. https://doi.org/10.1006/jare.2002.1003

Silva, D. E., Silva, G. L. D., Nascimento, J. M. D., Ferla, N. J. (2018) Mite fauna associated with bird nests in Southern Brazil. Systematic and Applied Acarology, 23, 426-440. http://dx.doi.org/10.11158/saa.23.3.2

Skvarla, M. J., Fisher, J. R., Dowling, A. P. G. (2014) A review of Cunaxidae (Acariformes, Trombidiformes): Histories and diagnoses of subfamilies and genera, keys to world species, and some new locality records. Zookeys, 418, 1-103. http://dx.doi.org/10.3897/zookeys.418.7629

Solarz, K., Szilman, P., Szilman, E., Krzak, M., Jagla, A. (2004) Some allergenic species of astigmatid mites (Acari, Acaridida) from different synanthropic environments in southern Poland. Acta Zoologica Cracoviensia, 47, 125-145. https://doi.org/10.3409/173491504783995843

Tryjanowski, P., Baraniak, E., Bajaczyk, R., Gwiazdowicz, D. J., Konwerski, S., Olszanowski, Z., Szymkowiak, P. (2001) Arthropods in nests of the red-backed shrike (Lanius collurio) in Poland. Belgian Journal of Zoology, 131, 69-74. 
Urhan, R., Katilmis, Y., Kahveci, A. O. (2008) Vertical distribution of soil mites (Acari) in Dalaman (Mugla Pro.-Turkey). Munis Entomology and Zoology Journal, 3, 333-341.

Vázquez-González, M. M. (2012) Informe final: Riqueza específica y biodiversidad de microartrópodos edáficos de la Isla de Cozumel, Q. Roo. Proyecto SNIB-CONABIO No. HJ028. Universidad de Quintana Roo, México, 52 pp.

Vieira de Souza, I. (2010). Phytoseiidae em fruteiras cultivadas e padrao de ocorrencia de Aceria guerreronis Keifer (Eriophyidae) e outros ácaros em frutos de coqueiro no Sul da Bahia. Universidae Estadual de Santa Cruz. Tesis Maestría. Brasil, 11 pp.

Walter, D. E., Lindquist, E. E., Smith, I. M., Cook, D. R., Krantz, G. W. (2009) Chapter thirteen: Order Trombidiformes. Pp. 233-420. In: Krantz, G. W., Walter, D. E. (Eds.). A manual of Acarology. Tech University Press, Texas.

Watson, J. (2010) The golden eagle. Second edition, Yale University Press, New Haven, Connecticut. United State of America, $448 \mathrm{pp}$.

Weigmann, G. (2013) Ecology and biogeography of oribatid mites (Acari: Oribatida) from the coastal region of Portugal. Soil organisms, 85, 147-160.

Wimberger, P. H. (1984) The use of green plant material in bird nests to avoid ectoparasites. Auk, 101, 615-618.

Zaher, M. A., Soliman, Z. R., El-Bishlawy, S. M. (1975) Feeding habits of the predaceous mite, Cunaxa capreolus (Acarina: Cunaxidae). Entomophaga, 20, 209-212. 